\title{
Large-Scale Integration of Human Genetic and Physical Maps
}

\author{
Caroline M. Nievergelt, ${ }^{1}$ Douglas W. Smith, ${ }^{1,2}$ J. Bradley Kohlenberg, ${ }^{1}$ and \\ Nicholas J. Schork ${ }^{1,3}$ \\ ${ }^{1}$ Polymorphism Research Laboratory, Department of Psychiatry and ${ }^{2}$ Department of Biology, University of California at San \\ Diego, La Jolla, California 92093-0603, USA
}

\begin{abstract}
Genetic maps are used routinely in family-based linkage studies to identify the rough location of genes that influence human traits and diseases. Unlike physical maps, genetic maps are based on the amount of recombination occurring between adjacent loci rather than the actual number of bases separating them. Genetic maps are constructed by statistically characterizing the number of crossovers observed in parental meioses leading to the transmission of alleles to their offspring. Considerations such as the number of meioses observed, the heterozygosity and physical distance between the loci studied, and the statistical methods used can impact the construction and reliability of a genetic map. As is well known, poorly constructed genetic maps can have adverse effects on linkage mapping studies. With the availability of sequence-based maps, as well as genetic maps generated by different researchers (such as those generated by the Marshfield and deCODE groups), one can investigate the compatibility and properties of different maps. We have integrated information from the most current human genome sequence data (UCSC genome assembly Human July 2003) as well as 8399 microsatellite markers used in the Marshfield and deCODE maps to reconcile the these maps. Our efforts resulted in updated sex-specific genetic maps.
\end{abstract}

[Supplemental material is available online at www.genome.org and http://elcapitan.ucsd.edu/hyper/.]

The use of genetic maps for identifying genes that influence human traits and diseases has a long and illustrious history in the medical and biological sciences. Indeed, as pointed out in a recent historical perspective on human biological research by Lander and Weinberg (2000), many advances in the identification of disease-predisposing genes for traits and diseases of all sorts have paralleled advances in the development of genetic and physical maps. Unlike physical maps of genomic positions or loci-which attempt merely to tally the number of bases separating adjacent loci-genetic maps seek to characterize the frequency of meiotic recombination occurring between loci. As such, the construction of genetic maps is largely a statistical undertaking, whereby individuals within families are genotyped at many loci and the patterns associated with the transmission of alleles are recorded to determine how often alleles or variants at adjacent loci are either coinherited or shuffled as a result of recombination in relevant parental meioses (see, e.g., Ott 1999).

The reconciliation of locus positions dictated by genetic and physical maps is not trivial, unfortunately. Large-scale sequencing and the ordering of loci from multiple sequence reads can result in errors of all sorts, most simply caused by sequencing errors and missing sequence or "gaps" between the sequences (see, e.g., Lander et al. 2001; Venter et al. 2001). These errors are often corrected over time, resulting in periodic updates to available human genome sequence information. There are also issues that compromise the reliability of genetic maps. As is well recognized and emphasized in the description of the recent construction of the genetic map by Kong et al. (2002), factors such as the number of meioses studied and the informativity of the DNA markers used, can impact the reliability of a genetic map. In addition, it is also known that the construction and ultimate use

\footnotetext{
${ }^{3}$ Corresponding author.

E-MAIL nschork@ucsd.edu; FAX (858) 822-2113.

Article and publication are at http://www.genome.org/cgi/doi/10.1101/ gr.1475304. Article published online before print in May 2004.
}

of genetic maps can be compromised because of missing data and simple genotyping errors (Goring and Terwilliger 2000).

As a result of problems associated with the construction of human physical and genetic maps, differences with respect to the positions of loci exist not only between physical and genetic maps, but also among different physical and genetic maps. Because genetic maps play a crucial role in pedigree-based meiotic (or "linkage") mapping gene discovery strategies, it is important to consider their reconcilability with physical maps. In addition, it has been well documented that misspecification of genetic maps can have negative effects on linkage analyses (see, e.g., Daw et al. 2000; Goring and Terwilliger 2000; Hackett and Broadfoot 2003). Three types of genetic map misspecification, listed from the most severe forms of misspecification to the least severe, plague linkage analyses, especially in the context of "multipoint" analyses that depend crucially on reliable DNA marker positions: (1) Loci are erroneously assumed to be on a particular chromosome. (2) Loci are out of order but on the correct chromosome. (3) Loci are on the correct chromosome and in the correct order but their interlocus distances, in terms of the amount of recombination that occurs between them, are misspecified. It must be emphasized that when reconciling genetic and physical maps, there are likely to be errors in each-despite the efforts of those who created them. Thus, incompatibilities found across the maps really only point out areas of the genome that call for reevaluation of both types of map.

Some researchers have considered the quality and reconcilability of genetic and physical maps (see http://cedar.genetics. soton.ac.uk/public_html/LDB2000), but most of these efforts have focused on a single chromosome or genomic region (see, e.g., Tapper et al. 2001; Matise et al. 2002), or were pursued prior to recent updates of the draft human genome sequence and the availability of the comprehensive genetic map constructed by Kong et al. (2002; see, e.g., Yu et al. 2001; DeWan et al. 2002). In this paper, we extend a recent study by Matise et al. (2002) that sought to evaluate the comparability of genetic and physical 
maps involving a subset of routinely used genetic markers on Chromosome 22, by considering the entire set of 8325 microsatellite (STRPs) markers examined by Broman et al. (1998; referred to as the "Marshfield" map and markers), the 5136 microsatellites examined in a recently developed genetic map by Kong et al. (2002; referred to as the "deCODE" map and markers), and the most recent releases of the sequence of the Human genome (UCSC genome assembly Human July 2003, http://genome.ucsc. edu/; and the Celera database, http://myscience.appliedbiosystems. com/; Venter et al. 2001). We document ambiguities and differences in the physical and genetic maps and comment on the problems these ambiguities might create when the maps are used for different purposes.

Most importantly, we have developed a comprehensive and integrated sex-specific genetic map that could be used in, for example, multipoint linkage analyses or help focus further sequencing and marker ordering efforts. Our map includes all of the Marshfield and deCODE markers and is based primarily on the genetic positions of loci in deCODE map because of the large number of meioses studied to develop that map. The highresolution deCODE map is based on 1257 meiotic events in twogeneration pedigrees and is the most accurate genetic map available to date. However, deCODE only includes 5136 microsatellites, whereas the older, less accurate Marshfield map, which was calculated based on only 188 meioses in three generation pedigrees, includes 8325 microsatellite markers. Our map uses up-to date physical sequence information to interpolate the positions of markers not included in the deCODE map, but whose position can be estimated relative to markers on that map. A method for querying markers in this map using a simple Excel spreadsheet query macro is available as Supplemental material from the authors' Web site (http://elcapitan.ucsd.edu/hyper/).

\section{RESULTS}

\section{Chromosome Positions}

Of the 7737 Marshfield markers with available physical positions (UCSC July 2003), 45 were found to have chromosomal assignments that did not match the assignment provided by the physical map (Table 1). Of these 45 markers, 28 are "Utah" markers, which were typed on only a few meioses (four of the eight CEPH families used to form the Marshfield genetic maps). Only one of these 45 markers was considered in the construction of the deCODE map. In addition, only three markers are used in the routine linkage mapping panels provided by Marshfield Clinic. Three markers (PLA2, GC, and FB7F11) have been mapped to the chromosome assigned by the physical map via radiation hybrids, suggesting that the initial chromosomal positions provided by the Marshfield map were likely wrong. Finally, some of these markers have chromosomal assignments based on the physical map that have changed as the physical map has been updated (Table 1, cf. columns 9-11), suggesting a lack of confidence in their location as dictated by available sequence information. For comparison purposes, we also provide positional information obtained from the Celera database for these 45 markers. For a subset of these markers, it is quite possible that the sequence-based position is simply wrong as well.

\section{Marker Order}

To assess marker order, we considered the number of markers implicated in a "block" of markers where there is misspecification. To clarify, consider a string of markers whose correct order is 1-2-3-4-5-6-7-8-9-10-11-12, but whose assumed order is $1-2-3-9-7-4-5-6-8-10-11-12$ : the block of markers 9-7-4-5$6-8$ is "order misspecified." This type of order misspecification captures entire blocks, such that if, say, multipoint linkage methods were used to extract identity-by-descent allele-sharing information among relative pairs, the calculations would be wrong even though some of the markers in the block are in the correct order (i.e., markers 4-5-6 are not out of order but do sit in a block that contains order misspecification). Table 2 describes the percentages of markers examined from the Marshfield and deCODE genetic maps exhibiting block order problems, the average and maximum block length on a chromosome-by-chromosome basis, and the percentage of markers that are misplaced in their position by $>2 \mathrm{cM}$ and $>5 \mathrm{cM}$, respectively. These parameters were calculated by ordering the markers according to their physical position and determining all markers that are out of order compared with their Marshfield and deCODE map locations, respectively. For these markers, "true" map positions were interpolated by using the physical and genetic map position of the markers preceding and following a block with misspecified markers, analogous to the way described in the Methods section for the comprehensive genetic map. This resulted in especially large values for Chromosomes 16 and 17 for the Marshfield map and for Chromosomes 5 and 7 for the deCODE map, respectively. These large values are partly caused by a few markers with relatively large disagreement between their physical and genetic map positions, which, in accordance with our definition of blocks, will result in a large maximum block length. Furthermore, because we used markers that were adjacent to the blocks as a basis for the estimation of the "true" genetic position of the markers in these blocks, a large block length negatively influences the accuracy of the estimated marker positions as well as the number of markers misplaced at a certain distance.

In general, markers whose order is misspecified on the genetic maps that reside in a small region of the genome are likely to arise from difficulties in resolving recombination events between markers with a finite number of meioses. Although it is clear that the deCODE map contains fewer order problems than the earlier Marshfield map, this is more than likely because Kong et al. (2002) had the luxury of leveraging human genome sequence information in the construction of their map, as well as having many more meiotic events. However, discrepancies in order between the deCODE map and the physical map do exist, and this probably reflects updates and changes to the physical map that occurred after Kong et al. (2002) constructed their map. In addition, as with markers on genetic maps with different chromosome positions from those provided by the physical map, there is the possibility that the physical map positions are incorrect as well and that the genetic orderings are correct in one, the other, or both of the genetic maps.

\section{Interlocus Distances}

To assess potential differences in the amount of recombination thought to occur between loci, we compared interlocus recombination fractions between marker loci across the Marshfield and deCODE maps. This comparison involved 4961 markers whose relative positions on all three maps could be obtained. Markers were first ordered according to their physical position, then the absolute genetic distance between adjacent markers was calculated for both the Marshfield and deCode maps. Differences in these interlocus distances between the two maps are shown in Figure 1. It can be seen that although there are some adjacent loci for which large discrepancies (i.e., $>5 \mathrm{cM}$ difference) in the amount of recombination exist, they are relatively few $(<3 \%)$. In fact, $59 \%$ of the interlocus distances between the two maps are between 1 and $2 \mathrm{cM}$. This is owing to the fact that we studied such a large number of markers with a density large enough to preclude big gaps between our markers whose relative positions could be reconciled with the physical map. Differences in recom-

\section{Genome Research}


Table 1. Microsatellite Markers ( $N=45)$ Whose Sequence Positions (UCSC Assembly Human July 2003/NCBI Build 34) Do Not Correspond to the Marshfield Chromosomal Position (http://research.marshfieldclinic.org/genetics/)

\begin{tabular}{|c|c|c|c|c|c|c|c|c|c|c|c|}
\hline \multirow{2}{*}{$\begin{array}{l}\text { Marker } \\
\text { name }\end{array}$} & \multicolumn{2}{|r|}{ UCSC } & \multirow{2}{*}{$\begin{array}{l}\text { Marshfield } \\
\text { chromosome }\end{array}$} & \multirow{2}{*}{$\begin{array}{c}\text { Other } \\
\text { genetic } \\
\text { maps }\end{array}$} & \multirow{2}{*}{$\begin{array}{c}\text { Yac map } \\
\text { chromosome }\end{array}$} & \multirow{2}{*}{$\begin{array}{c}\text { RH maps } \\
\text { chromosome }\end{array}$} & \multirow{2}{*}{$\begin{array}{l}\text { Marshfield } \\
\text { screening }\end{array}$} & \multicolumn{3}{|c|}{ UCSC } & \multirow{2}{*}{$\begin{array}{c}\text { CELERA } \\
03 / 03\end{array}$} \\
\hline & ID & Chromosome & & & & & & $11 / 02$ & $06 / 02$ & $04 / 02$ & \\
\hline UT5086 & 7782 & 1 & 9 & No & No & No & No & 1 & 1 & 1 & No \\
\hline PLA2 & 7128 & 1 & 12 & No & No & Gb4, G3, Wi:1 & No & 1 & 1 & 1 & 1 CelAnno \\
\hline GC/SGC31591 & 5929 & 2 & 4 & No & No & Wi:2 & No & 2 & 2 & 2 & 2RefSeq \\
\hline UT710 & 6472 & 2 & 7 & No & No & No & No & 2 & 2 & 2 & No \\
\hline UT1307 & 7844 & 2 & 20 & No & No & No & 10 & 2 & 2 & 2 & 2 RefSeq \\
\hline AFMA101XG5 & 842 & 3 & 2 & Gen:2 & No & No & No & 3 & 3 & 3 & No \\
\hline GATA43F06 & 5557 & 3 & 2 & No & Wi:3 & No & No & 3 & 3 & 3 & No \\
\hline UT885 & 6629 & 3 & 8 & No & No & No & No & 3 & 3 & 3 & No \\
\hline UT5819 & 6876 & 3 & 10 & No & No & No & No & 3 & 3 & 3 & No \\
\hline ATA4F06 & 6032 & 4 & 5 & No & No & No & $4-9$ & 4 & No & 4 & No \\
\hline UT1531 & 6710 & 4 & 9 & No & No & No & No & 4 & 4 & 4 & No \\
\hline UT2361 & 6715 & 5 & 9 & No & No & No & No & 5 & 5 & 5 & No \\
\hline AFM023TG5 & 4343 & 5 & 17 & Gen:17 & No & No & No & 5 & 5 & 5 & 17 RefSeq \\
\hline ATA1H07 & 7502 & 5 & 17 & No & No & No & No & 5 & 5 & 5 & 5 CelAnno \\
\hline GATA124A11 & 7816 & 6 & 20 & No & No & No & No & 6 & No & No & 6 CelAnno \\
\hline GATA116E08 & 7893 & 8 & 21 & No & No & No & 6 & 8 & 8 & 8 & No \\
\hline AFM123ус5 & 328 & 8 & 1 & Gen:1 & No & No & No & 8 & No & 8 & No \\
\hline UT924 & 7349 & 10 & 14 & deC:14 & No & No & No & 10 & 10 & 10 & No \\
\hline ATA37G10 & 6499 & 11 & 8 & No & No & No & No & 11 & 11 & 11 & No \\
\hline UT1607 & 6989 & 12 & 11 & No & No & No & No & 12 & 12 & 12 & No \\
\hline UT935 & 7414 & 12 & 15 & No & No & No & No & 12 & 12 & 12 & No \\
\hline UT1888 & 7327 & 16 & 14 & No & No & No & No & 16 & 16 & 22 & 1 RefSeq \\
\hline UT1598 & 7551 & 16 & 17 & No & No & No & No & 16 & 16 & 16 & No \\
\hline UT1025 & 7911 & 16 & 21 & No & No & No & No & 16 & No & No & No \\
\hline UT556 & 7920 & 16 & 21 & No & No & No & No & 16 & No & 2,13 & No \\
\hline GAAT1A12 & 6043 & 16 & 5 & No & Wi:5 & No & No & $16(2 \times)$ & 16 & 5 & No \\
\hline UT18 & 5621 & 17 & 2 & No & No & No & No & 17 & 17 & 17 & No \\
\hline UT5182 & 6611 & 18 & 8 & No & No & No & No & 18 & 18 & 18 & 18 RefSeq \\
\hline UT5146 & 5641 & 21 & 2 & No & No & No & No & 11 & 1,2 & 1 & No \\
\hline GATA115F05 & 6519 & 21 & 8 & No & No & No & No & 21 & 21 & 21 & 21 RefSeq \\
\hline UT5116III & 5638 & 22 & 2 & No & No & No & No & 22 & 22 & 14,22 & No \\
\hline UT597 & 7339 & 22 & 14 & No & No & No & No & 22 & 14,22 & 14,22 & No \\
\hline UT6047 & 7341 & 22 & 14 & No & No & No & No & 22 & No & $2,14,22$ & No \\
\hline UT7691 & 5657 & $X$ & 2 & No & No & No & No & $X$ & No & No & 2 CelAnno \\
\hline UT764 & 7635 & $x$ & 9 & No & No & No & No & $x$ & $X$ & $x$ & 1 RefSeq \\
\hline UT832 & 7020 & $x$ & 11 & No & No & No & No & $x$ & No & No & X CelAnno \\
\hline UT6574 & 7156 & $x$ & 12 & No & No & No & No & $x$ & $X$ & $X$ & No \\
\hline ATA5E03 & 5293 & Y & 1 & No & No & No & 7 & $\mathrm{Y}$ & $\mathrm{Y}$ & Y & No \\
\hline IFNAR & 7908 & Y & 21 & No & No & No & No & $Y$ & Y & $1, Y$ & No \\
\hline
\end{tabular}

A comparison to chromosomal locations from additional sources is shown.

Key: 7737 markers are included in the comparison. Available positional information from genetic maps (Genethon, DeCODE), Yac map (Whitehead Institute), and radiation hybridization (RH) maps (NCBI GeneMap99 GB4 and G3, Whitehead Institute, and Stanford TNG RH maps) is provided to help determine which source of information is erroneous. In addition, markers included in the commonly used Marshfield screening sets (1-12) are indicated. Chromosomal positions are also compared with older UCSC assemblies and with the sequence-based Celera database (http:// myscience.appliedbiosystems.com/). Markers listed on the Y-chromosome are in the pseudoautosomal region of the Y-chromosome.

bination rates between the same loci in the two genetic maps could be the result of errors in positioning the markers, sampling variation due to the finite number of meioses used to construct each map, or inherent differences in recombination rates in the populations used to construct the maps.

\section{Comprehensive Genetic Map}

From our efforts at examining the reconcilability and comparability of the Marshfield and deCODE genetic maps along with the latest versions of the available human genome sequence, we developed a comprehensive and integrated map (see Methods). An extract is shown in Table 3. The comprehensive genetic map includes a total of 8399 markers with information on the physical position (UCSC assembly July 2003), and Marshfield and deCODE recombination rates, as well as interpolated deCODE values for 2838 markers. A summary is shown in Table 4. This map is available for downloading with macros to facilitate marker searches from http://elcapitan.ucsd.edu/hyper/. 
Table 2. Number and Percentage of Microsatellites, Block Length, and Percentage of Markers Misplaced for the 7634 Marshfield and 5093 deCODE Markers With Unambiguously Determined Physical Positions Whose Marker Order (See Text) Does Not Correspond to the Order Based on the Sequencing Data (UCSC Assembly July 2003; NCBI Build 34)

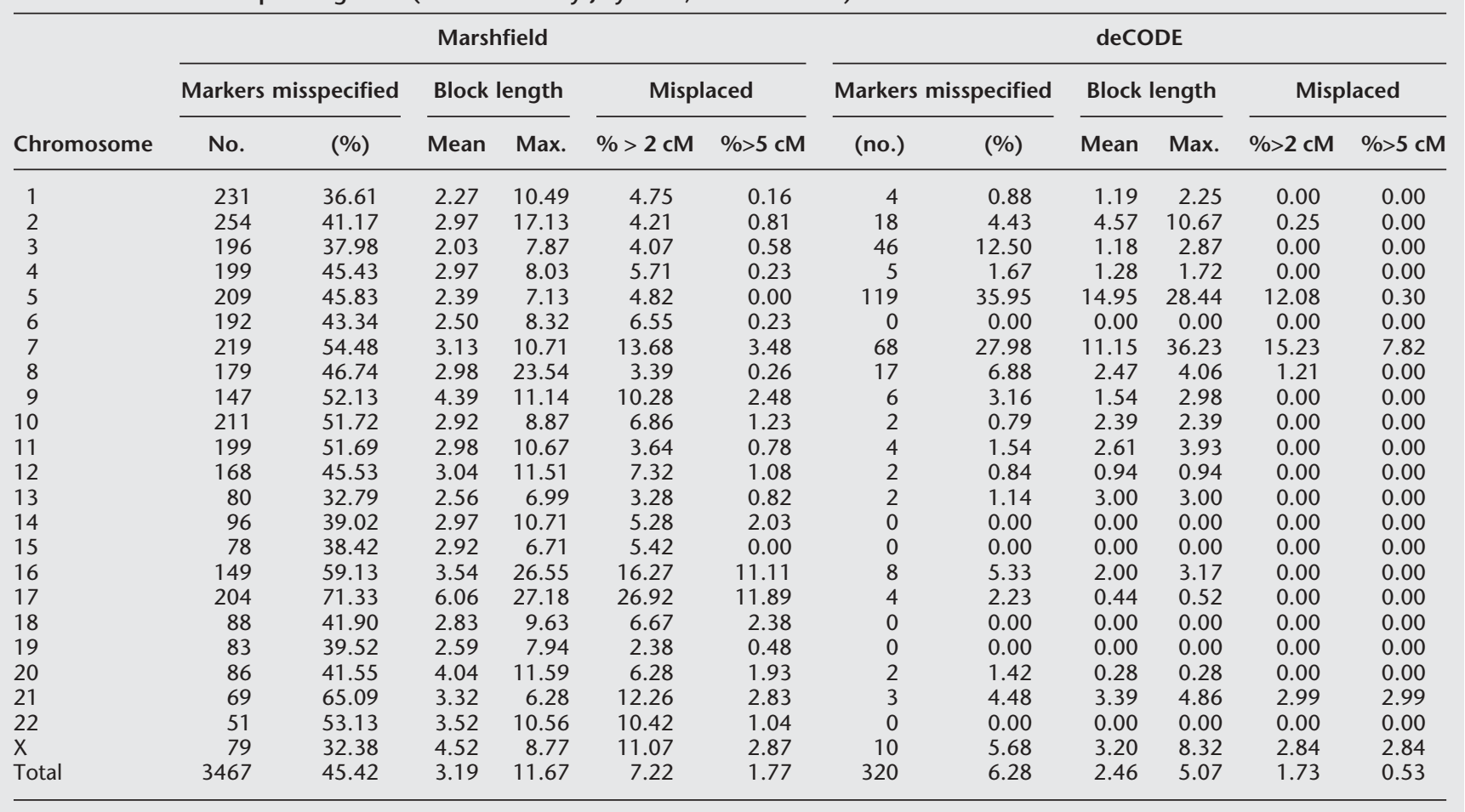

\section{DISCUSSION}

The study of recombination rates, as well as the exploitation of recombination rates in human gene-mapping studies, have received a great deal of attention, especially since the publication

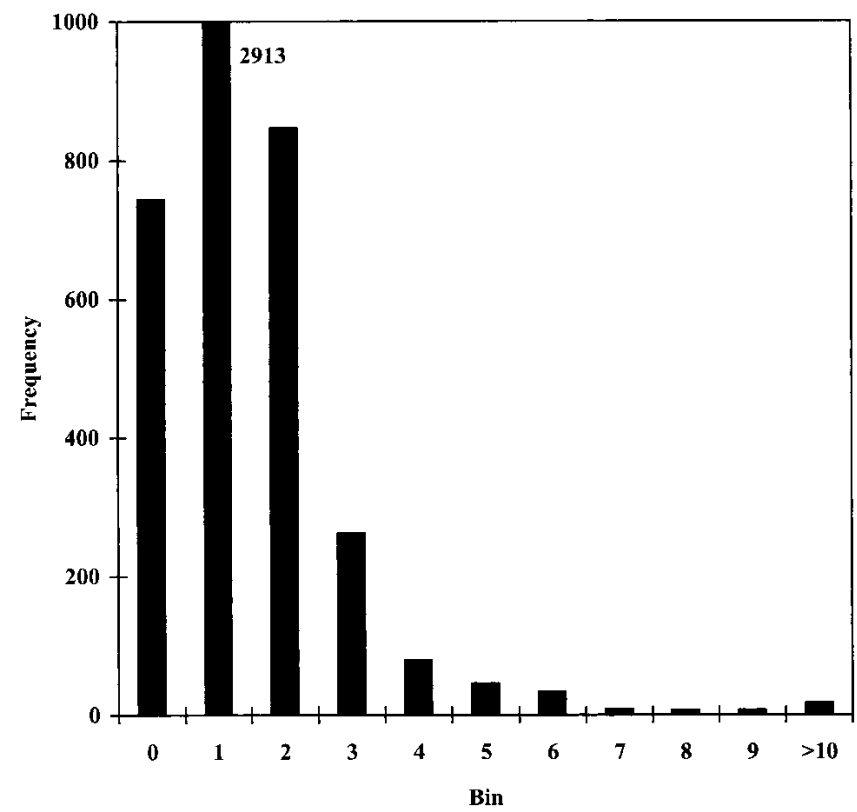

Figure 1 Distribution of the differences in interlocus distances between 4961 markers quoted both in the initial Marshfield map (Broman et al. 1998) and also computed in the newer deCODE map (Kong et al. 2002). Note: Bin >10 includes interlocus distance differences up to $36 \mathrm{cM}$. of the draft of human genome sequence and the announcement of the human Haplotype Map Initiative (see, e.g., Dawson et al. 2002; Gabriel et al. 2002). With the current availability of multiple physical and genetic maps, it behooves researchers to consider their reconcilability, if for no other reason than to have confidence in their correctness. Our assessment of the complete set of markers used in the construction of the genetic maps by Broman et al. (1998) and Kong et al. (2002) in conjunction with an updated physical map suggests that many discrepancies in maps exist, raising the question of which map might be correct. Although it is quite likely that the genetic map constructed by Kong et al. (2002) is more reliable then the initial Broman et al. (1998) map, given the use of a larger number of meioses in the construction of that map and the fact that Kong et al (2002) pursued the construction of their map after the publication of the draft human genome sequence, there still may be problems with all maps. The published human genome sequence is somewhat "fluid" given constant updating, and this is consistent with the existence of discrepancies involving the Kong et al. (2002) map compared with the physical map, as we have shown.

Map misspecification can have serious negative consequences on gene-mapping studies. Incorrect physical maps can complicate late stages of positional cloning efforts, whereas incorrect genetic maps can complicate initial linkage analyses. Our analysis involved all available markers in the Marshfield (Broman et al. 1998) and deCODE maps (Kong et al. 2002) and not just the few hundred or so that researchers might use in an initial linkagebased gene-mapping project. Thus, our findings relating to marker order involved many more markers (and hence many more opportunities for marker order misspecification) than is likely to arise in a routine linkage analysis. Despite this, we have found evidence for map problems with previous linkage analyses using specific sets of DNA markers. For example, in our reassess-

\section{Genome Research}


Table 3. Extract From the "Comprehensive Genetic Map" Available on the Author's Web Page, Showing UCSC Physical Positions and Average (av.), Female (f), and Male (m) Recombination Rates (in Centimorgans) From the deCODE (deC) and Marshfield (M) Genetic Maps

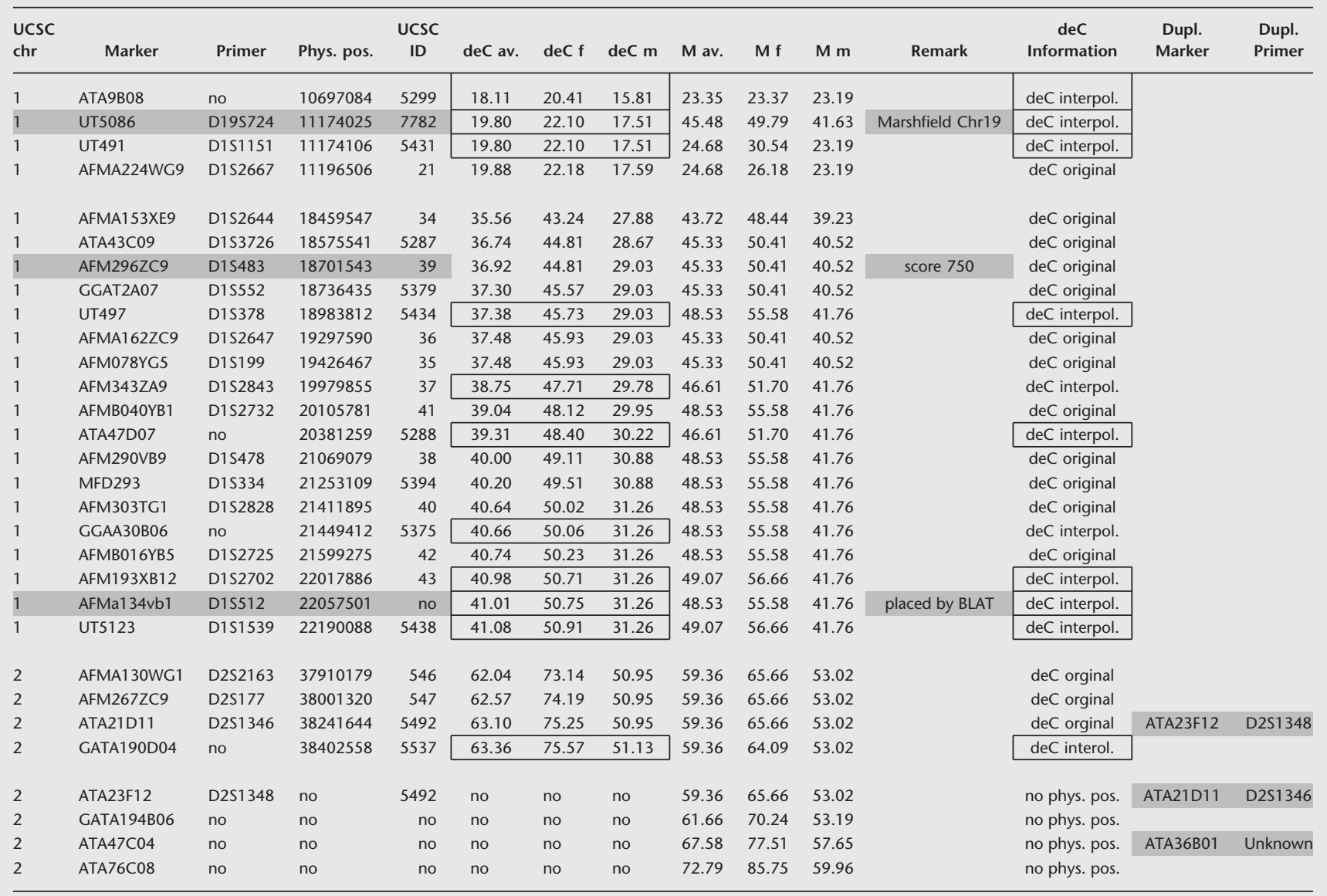

Missing genetic positions for the deCODE map were interpolated (see Methods).

ment of linkage analyses of autism using data available in the public domain (data available from http://www.agre.org), we identified a marker (UT1307) that was initially thought to be on Chromosome 20 but has since been physically mapped to Chromosome 2 (C. Mathews, C. Nievergelt, and N. Schork, in prep.). Subsequent genetic analyses of markers on Chromosomes 2 and 20 showed that the marker in question, indeed, belonged to a linkage group on Chromosome 2. Although our reanalyses of linkages to autism on Chromosomes 2 and 20 did not result in evidence for linkage, the potential was there for initial reports to have not found evidence for linkage because the multipoint procedures they used were, in some sense, infected by a DNA marker locus whose alleles must have provided erroneous recombination patterns with neighboring marker allele patterns. We also identified some differences in interlocus distances between markers used in that map and interlocus distances computed by us.

One easy way potentially to verify that the genetic map used for a particular linkage analysis study is reliable is to assess the linkages between the markers empirically using the family data at hand (i.e., merely assess linkages among the markers using programs such as CRI-MAP or ASPEX rather than linkages between the markers and a potential trait-influencing locus). One can then compare the assumed map with that derived from the data. Although published genetic maps such as the Marshfield map with 188 meioses in three generation pedigrees and the deCODE map with 1257 meioses in two-generation families might be based on more meiotic events than those available in a given linkage study, such an exercise is well worth the effort as it can reveal other items in addition to incorrect map positions such as genotyping errors.

In pursuing empirical studies of map reliability with a given data set, it may be worthwhile to consider the use of sex-specific recombination rates. It is well known that males and females differ greatly in recombination rates (Broman et al. 1998; Kong et al. 2002). Although the construction of sex-specific maps may be less reliable than constructing a sex-averaged map, because the number of meioses in a given data set will be less (usually half) for either sex in isolation, the proper implementation of multipoint linkage analysis requires correct specification of recombination rates (Daw et al. 2000; see also Schork and Greenwood 2004). In this light, it is rather awkward that many of the most widely used programs for human linkage analysis such as GENEHUNTER (Kruglyak et al. 1996), SOLAR (Almasy and Blangero 1998), and MERLIN (Abecasis et al. 2002) do not accommodate sex-specific maps in their implementation of multipoint linkage analysis.

Discrepancies between genetic and physical maps will likely diminish as more and more polymorphic loci are mapped. In addition, further genetic linkage analyses of DNA markers (e.g., those used in genetic mapping studies) will provide greater con- 
Nievergelt et al.

Table 4. Summary Information of the "Comprehensive Genetic Map"

\begin{tabular}{|c|c|c|c|c|c|c|c|c|c|c|c|}
\hline \multirow[b]{3}{*}{ Chromosome } & \multirow{3}{*}{$\begin{array}{c}\text { Physical } \\
\text { distance spanned } \\
\text { (kb) }\end{array}$} & \multicolumn{3}{|c|}{ Marshfield } & \multicolumn{3}{|c|}{ deCODE original and interpolated } & \multirow{2}{*}{\multicolumn{4}{|c|}{$\begin{array}{l}\text { Gaps where markers were } \\
\text { interpolated }(\mathrm{cM}) \text { and }(\mathrm{kb})\end{array}$}} \\
\hline & & \multirow{2}{*}{$\begin{array}{l}\text { No. of } \\
\text { markers }\end{array}$} & \multirow{2}{*}{$\begin{array}{l}\text { Distance } \\
\text { spanned } \\
\text { (cM) }\end{array}$} & \multirow{2}{*}{$\begin{array}{c}\text { Average } \\
\text { spacing } \\
\text { (cM) }\end{array}$} & \multirow{2}{*}{$\begin{array}{l}\text { No. of } \\
\text { markers }\end{array}$} & \multirow{2}{*}{$\begin{array}{l}\text { Distance } \\
\text { spanned } \\
\text { (cM) }\end{array}$} & \multirow{2}{*}{$\begin{array}{l}\text { Average } \\
\text { spacing } \\
\text { (cM) }\end{array}$} & & & & \\
\hline & & & & & & & & Mean & Max. & Mean & Max. \\
\hline 1 & 245,963 & 687 & 289.66 & 0.42 & 667 & 276.40 & 0.42 & 0.87 & 4.97 & 969 & 23,665 \\
\hline 2 & 243,243 & 657 & 269.07 & 0.41 & 635 & 266.79 & 0.42 & 1.03 & 4.23 & 1087 & 9342 \\
\hline 3 & 199,130 & 549 & 231.27 & 0.42 & 548 & 221.83 & 0.40 & 0.96 & 5.03 & 859 & 3975 \\
\hline 4 & 191,136 & 466 & 211.65 & 0.46 & 456 & 204.74 & 0.45 & 1.03 & 6.5 & 983 & 4185 \\
\hline 5 & 180,458 & 493 & 197.54 & 0.40 & 479 & 205.69 & 0.43 & 1.13 & 7.47 & 1016 & 5204 \\
\hline 6 & 170,695 & 480 & 193.14 & 0.40 & 465 & 189.60 & 0.41 & 1.07 & 6.43 & 961 & 6232 \\
\hline 7 & 157,015 & 440 & 181.97 & 0.41 & 428 & 187.09 & 0.43 & 1.93 & 36.23 & 1042 & 4686 \\
\hline 8 & 146,149 & 408 & 194.32 & 0.48 & 399 & 166.08 & 0.42 & 1.19 & 6.19 & 1117 & 5106 \\
\hline 9 & 136,236 & 309 & 168.98 & 0.55 & 298 & 160.01 & 0.54 & 1.12 & 5.45 & 1054 & 5177 \\
\hline 10 & 134,677 & 440 & 173.13 & 0.39 & 420 & 179.12 & 0.42 & 1.14 & 5.11 & 868 & 3677 \\
\hline 11 & 133,704 & 404 & 147.77 & 0.37 & 398 & 152.45 & 0.38 & 0.95 & 7.1 & 841 & 4590 \\
\hline 12 & 131,939 & 404 & 170.66 & 0.42 & 391 & 171.96 & 0.44 & 1.09 & 4.5 & 928 & 6007 \\
\hline 13 & 112,931 & 255 & 114.98 & 0.45 & 250 & 129.52 & 0.52 & 1.25 & 5.05 & 896 & 2698 \\
\hline 14 & 103,950 & 264 & 138.18 & 0.50 & 251 & 124.81 & 0.48 & 1.13 & 5.87 & 763 & 2183 \\
\hline 15 & 100,070 & 213 & 122.14 & 0.58 & 203 & 133.61 & 0.66 & 1.55 & 8.29 & 1089 & 2972 \\
\hline 16 & 89,242 & 276 & 134.12 & 0.49 & 266 & 130.00 & 0.49 & 1.37 & 7.3 & 1009 & 15,264 \\
\hline 17 & 81,287 & 313 & 126.46 & 0.41 & 297 & 137.99 & 0.46 & 1.16 & 5.75 & 716 & 4534 \\
\hline 18 & 75,963 & 231 & 126.00 & 0.55 & 218 & 121.65 & 0.56 & 1.17 & 4.12 & 830 & 4231 \\
\hline 19 & 63,727 & 237 & 105.02 & 0.45 & 218 & 109.73 & 0.51 & 1.42 & 7.58 & 876 & 10,696 \\
\hline 20 & 59,563 & 228 & 101.22 & 0.45 & 220 & 98.63 & 0.45 & 0.99 & 3.97 & 722 & 5060 \\
\hline 21 & 46,894 & 114 & 61.40 & 0.54 & 114 & 78.48 & 0.66 & 1.84 & 7.42 & 829 & 1722 \\
\hline 22 & 48,058 & 107 & 142.83 & 1.35 & 103 & 70.60 & 0.69 & 1.82 & 8.88 & 1101 & 3616 \\
\hline$x$ & 153,510 & 271 & 206.74 & 0.77 & 261 & 190.67 & 0.73 & 1.68 & 6.51 & 1352 & 5345 \\
\hline Total & $3,005,542$ & 8246 & 3808 & 0.51 & 7985 & 3707 & 0.49 & 1.26 & 7.39 & 953 & 6094 \\
\hline
\end{tabular}

The distances spanned by the physical, Marshfield, and deCode map (including markers with interpolated recombination values), number of markers and mean marker spacing for the genetic maps, and mean and maximum gaps between original deCODE recombination rates where markers were interpolated are shown for all chromosomes.

fidence in marker order and interlocus distances. The genetic map that resulted from our own efforts encompasses physi$\mathrm{cal} /$ sequence information to interpolate positions of markers that are not available on either of the Marshfield or deCODE maps and as such is likely to be more comprehensive and accurate than either of them alone. Also, as the Haplotype Map Initiative unfolds, greater insight into recombination hot spots will emerge that can be further reconciled with different sorts of maps.

One final note on the issue of genetic and physical maps concerns their ubiquity. It is assumed that genetic maps capture recombination rates that are somewhat universal in that chromosomes are organized and recombined in roughly the same way among individuals. This assumed ubiquity is, in fact, what motivates researchers pursuing linkage analyses to use publicly available genetic maps in the first place. In addition, the potential ubiquity of the "block" structure of the human genome, which is motivating the human Haplotype Map Initiative, also assumes that, for example, genomic recombination hot spots, chromosomal sites for heavy gene conversion, and mutation hot spots are universal (see, e.g., Phillips et al. 2003; T. Greenwood, B. Rana, and N. Schork, in prep.). However, there is growing evidence that there exists very large variation in recombination rates among individuals during human meiosis (Cullen et al. 2002; Lynn et al. 2002). Because this individual variation is not really captured by simple specification of interlocus recombination fractions in genetic maps (which represent only "average" rates of recombination), it is an open question as to the degree to which such individual variation influences not only genetic map construction, but also the use of genetic maps in multipoint linkage analysis contexts.

\section{METHODS}

\section{Integration of Genetic and Physical Map Information}

To construct an "integrated map" including the newest physical positions for microsatellite markers used in the Marshfield (Broman et al. 1998; http://research.marshfieldclinic.org/genetics/) and deCODE maps (Kong et al. 2002), as well as sex-averaged and sex-specific recombination rates calculated in the two studies, we used information on physical positions from the University of California-Santa Cruz genome browser (July 2003 freeze; http:// genome.ucsc.edu/). Specifically, we used the 7879 physically positioned STS markers from UCSC that had a Marshfield and/or deCODE genetic map position. To compare markers from UCSC with deCODE and Marshfield, UCSC names were replaced with marker and primer names (i.e., D-numbers) used by the genetic maps (by looking them up on the genome database, UniSTS, etc.). The UCSC map was then complemented with sex-specific recombination rates from the two genetic maps. Finally, 42 markers (corresponding to 63 entries) with multiple physical positions (i.e., UCSC score <1000) and 45 markers whose chromosomal position on the UCSC map did not correspond to the position assigned by the genetic maps (see Table 1) were excluded. The resulting unambiguous "integrated map" with 7770 markers was used for all the analyses presented in this paper.

\section{Constructing a New "Comprehensive Genetic Map"}

To construct a "comprehensive genetic map" based on deCODE's genetic positions, we first ordered the 7770 markers included in the integrated map according to their UCSC physical positions and compared the marker order with its order on the deCODE map. Then 65 markers with order problems were excluded. Second, we estimated sex-specific and sex-averaged genetic positions (i.e., estimated deCODE positions) for the 2677 markers not used

\section{Genome Research}


in the deCODE map through linear interpolation between physical positions and genetic positions of adjacent deCODE markers, using the 5028 deCODE markers with corresponding orders between the physical and genetic maps. For example, a marker at a physical position of 50,000 bp would be placed two-thirds between two markers with original deCODE positions of $2 \mathrm{cM}$ at $10,000 \mathrm{bp}$ and $5 \mathrm{cM}$ at 70,000 bp, respectively, at an estimated genetic position of $4 \mathrm{cM}$. The use of linear interpolation of this sort is problematic for large interlocus distances, but not likely to be a huge problem for loci with small interlocus distances. Third, we included 88 markers with ambiguous physical positions (109 entries, see above) and 91 Marshfield and deCODE markers not placed on the UCSC July 2003 assembly, but whose physical positions could be found using BLAT (NCBI Entrez was used to find the relevant nucleotide sequence for a given STS, using annotation either from the nucleotide database or from the UniSTS database, and a FASTA-formatted version of the STS-containing sequence was then run at the BLAT facility of the UCSC Genome Center, to determine the chromosome and chromosome position of the marker in the UCSC coordinate system). Thus, 161 missing deCODE values of these markers were estimated as described above. Finally, 18 deCODE and 224 Marshfield markers without physical positions, as well as 187 cryptic duplicates from the Marshfield site, were added, resulting in a map with 8399 searchable marker entries. This comprehensive genetic map (see Table 3) is available as an Excel worksheet from the authors' Web site (http://elcapitan.ucsd.edu/hyper/), and includes a Macro for convenient Marker and Primer name look-up.

\section{ACKNOWLEDGMENTS}

This work was supported in part by the following NIH grants: the NHLBI Family Blood Pressure Program (FBPP; HL64777-01); the NHLBI hypertension SCOR program (HL54998); the NIH Pharmacogenetics Network (HL69758-01); and The Consortium on the Genetics of Schizophrenia (COGS; MH06557-01A1). The authors thank Tiffany Greenwood, John Kelsoe, and Daniel $\mathrm{O}^{\prime}$ Connor for critical discussions of this work.

The publication costs of this article were defrayed in part by payment of page charges. This article must therefore be hereby marked "advertisement" in accordance with 18 USC section 1734 solely to indicate this fact.

\section{REFERENCES}

Abecasis, G.R., Cherny, S.S., Cookson, W.O., and Cardon, L.R. 2002. MERLIN-Rapid analysis of dense genetic maps using sparse gene flow trees. Nat. Genet. 30: 97-101.

Almasy, L. and Blangero, J. 1998. Multipoint quantitative-trait linkage analysis in general pedigrees. Am. J. Hum. Genet. 62: 1198-1211.

Broman, K.W., Murray, J.C., Sheffield, V.C., White, R.L., and Weber, J.L. 1998. Comprehensive human genetic maps: Individual and sex-specific variation in recombination. Am. J. Hum. Genet. 63: 861-869.

Cullen, M., Perfetto, S.P., Klitz, W., Nelson, G., and Carrington, M. 2002. High-resolution patterns of meiotic recombination across the human major histocompatibility complex. Am. J. Hum. Genet. 71: 759-776.

Daw, E.W., Thompson, E.A., and Wijsman, E.M. 2000. Bias in multipoint linkage analysis arising from map misspecification. Genet. Epidemiol. 19: 366-380.

Dawson, E., Abecasis, G.R., Bumpstead, S., Chen, Y., Hunt, S., Beare, D.M., Pabial, J., Dibling, T., Tinsley, E., Kirby, S., et al. 2002. A first-generation linkage disequilibrium map of human chromosome
22. Nature 418: $544-548$

DeWan, A.T., Parrado, A.R., Matise, T.C., and Leal, S.M. 2002. The map problem: A comparison of genetic and sequence-based physical maps. Am. J. Hum. Genet. 70: 101-107.

Gabriel, S.B., Schaffner, S.F., Nguyen, H., Moore, J.M., Roy, J., Blumenstiel, B., Higgins, J., DeFelice, M., Lochner, A., Faggart, M., et al. 2002. The structure of haplotype blocks in the human genome. Science 296: 2225-2229.

Goring, H.H. and Terwilliger, J.D. 2000. Linkage analysis in the presence of errors III: Marker loci and their map as nuisance parameters. Am. J. Hum. Genet. 66: 1298-1309.

Hackett, C.A. and Broadfoot, L.B. 2003. Effects of genotyping errors, missing values and segregation distortion in molecular marker data on the construction of linkage maps. Heredity 90: 33-38.

Kong, A., Gudbjartsson, D.F., Sainz, J., Jonsdottir, G.M., Gudjonsson, S.A., Richardsson, B., Sigurdardottir, S., Barnard, J., Hallbeck, B. Masson, G., et al. 2002. A high-resolution recombination map of the human genome. Nat. Genet. 31: 241-247.

Kruglyak, L., Daly, M.J., Reeve-Daly, M.P., and Lander, E.S. 1996. Parametric and nonparametric linkage analysis: A unified multipoint approach. Am. J. Hum. Genet. 58: 1347-1363.

Lander, E.S. and Weinberg, R.A. 2000. Genomics: Journey to the center of biology. Science 287: 1777-1782.

Lander, E.S., Linton, L.M., Birren, B., Nusbaum, C., Zody, M.C., Baldwin, J., Devon, K., Dewar, K., Doyle, M., FitzHugh, W., et al. 2001. Initial sequencing and analysis of the human genome. Nature 409: 860-921.

Lynn, A., Koehler, K.E., Judis, L., Chan, E.R., Cherry, J.P., Schwartz, S., Seftel, A., Hunt, P.A., and Hassold, T.J. 2002. Covariation of synaptonemal complex length and mammalian meiotic exchange rates. Science 296: 2222-2225.

Matise, T.C., Porter, C.J., Buyske, S., Cuttichia, A.J., Sulman, E.P., and White, P.S. 2002. Systematic evaluation of map quality: Human chromosome 22. Am. J. Hum. Genet. 70: 1398-1410.

Ott, J. 1999. Analysis of human genetic linkage. The Johns Hopkins University Press, Baltimore, MD.

Phillips, M.S., Lawrence, R., Sachidanandam, R., Morris, A.P., Balding, D.J., Donaldson, M.A., Studebaker, J.F., Ankener, W.M., Alfisi, S.V., Kuo, F.S., et al. 2003. Chromosome-wide distribution of haplotype blocks and the role of recombination hot spots. Nat. Genet. 33: $382-387$

Schork, N.J. and Greenwood, T.A. 2004. Inherent bias toward the null hypothesis in conventional multipoint nonparametric linkage analysis. Am. J. Hum. Genet. 74: 306-316.

Tapper, W.J., Morton, N.E., Dunham, I., Ke, X., and Collins, A. 2001. A sequence-based integrated map of chromosome 22. Genome Res. 11: $1290-1295$.

Venter, J.C., Adams, M.D., Myers, E.W., Li, P.W., Mural, R.J., Sutton, G.G., Smith, H.O., Yandell, M., Evans, C.A., Holt, R.A., et al. 2001. The sequence of the human genome. Science 291: 1304-1351.

Yu, A., Zhao, C., Fan, Y., Jang, W., Mungall, A.J., Deloukas, P., Olsen, A., Doggett, N.A., Ghebranious, N., Broman, K.W., et al. 2001. Comparison of human genetic and sequence-based physical maps. Nature 409: 951-953.

\section{WEB SITE REFERENCES}

http://cedar.genetics.soton.ac.uk/public_html/LDB2000; The New Genetic Location Database.

http://elcapitan.ucsd.edu/hyper,/; NIH hypertension PPG home page. http://genome.ucsc.edu/; UCSC Genome Bioinformatics Home.

http://myscience.appliedbiosystems.com/; Celera Discovery System.

http://research.marshfieldclinic.org/genetics/; Welcome to the Center for Medical Genetics.

http://www.agre.org; Autism Genetic Resource Exchange.

Received April 28, 2003; accepted in revised form January 14, 2004.
Genome Research www.genome.org 


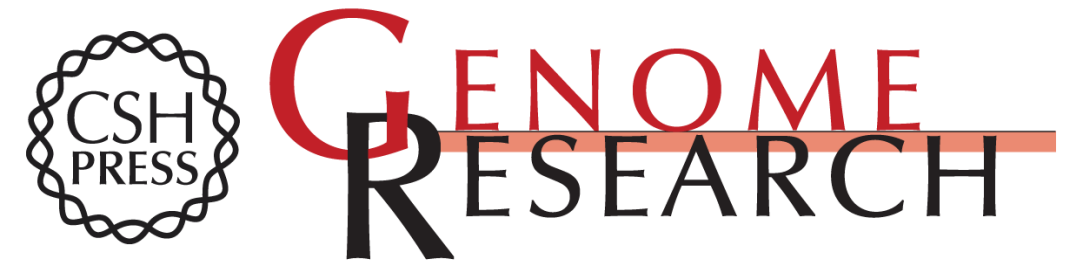

\section{Large-Scale Integration of Human Genetic and Physical Maps}

Caroline M. Nievergelt, Douglas W. Smith, J. Bradley Kohlenberg, et al.

Genome Res. 2004 14: 1199-1205

Access the most recent version at doi:10.1101/gr.1475304

Supplemental http://genome.cshlp.org/content/suppl/2004/05/13/1475304.DC1
Material

References This article cites 21 articles, 5 of which can be accessed free at: http://genome.cshlp.org/content/14/6/1199.full.html\#ref-list-1

License

Email Alerting

Receive free email alerts when new articles cite this article - sign up in the box at the top Service right corner of the article or click here.

\section{Affordable, Accurate} Sequencing.

To subscribe to Genome Research go to: https://genome.cshlp.org/subscriptions 